Vietnam Journal of Mechanics, NCST of Vietnam Vol. 25, 2003, No 4 (195-200)

\title{
FREQUENCY OF A VANDERPOL OSCILLATOR WITH LARGE CUBIC RESTORING NONLINEARITY
}

\author{
Nguyen Van Dinh and Tran Duong TRI \\ Institute of Mechanics, NCST Vietnam
}

In [2], a modified Poincaré method has been presented and applied to evaluate the period (frequency) of free oscillation in an undamped Duffing oscillator with large cubic restoring nonlinearity. There, the system examined is supposed to be near certain linear one with unknown (to be evaluated) frequency.

In this article, the same modified method is used to study the case of a Vanderpol oscillator: the self-excitation is assumed to be weak while the cubic restoring nonlinearity is assumed to be strong enough. It is shown that stationary self-excited oscillation nearly retains its amplitude while its frequency strongly depends on the cubic nonlinearity. The frequency obtained from the standard Poincaré method can only be used for small cubic nonlinearity; that obtained from the modified method is practically acceptable even for large cubic nonlinearity.

\section{Systems under consideration. Original amplitude and frequency}

Consider a Vanderpol oscillator described by the differential equation

$$
\ddot{x}+x=-\gamma x^{3}+h\left(1-x^{2}\right) \dot{x},
$$

where $x$ is oscillatory variable; overdots denote differentiation with respect to time $t ; \gamma>0$ is coefficient of the cubic restoring nonlinearity which may not be small; $h>0$ is a small intensity of the self-excitation $h\left(1-x^{2}\right) \dot{x}$.

For $\gamma=0$ (without cubic nonlinearity), in the first approximation, stationary self-excited oscillation is a stable harmonic one

$$
x=a \cos \psi
$$

with amplitude $a=2$ and with frequency $\omega=\dot{\psi}=1$. For small $\gamma$, the amplitude and the frequency are lightly modified. The question to be answered is how the amplitude and the frequency depend on the cubic nonlinearity if the later is strong enough.

\section{Frequency from the standard Poincaré method}

For the sake of comparison, the standard Poincaré method [1] is used first to evaluate the frequency in the case of small $\gamma$. 
Introducing the new dimensionless time $\tau=\omega t$, we rewrite the differential equation (1.1) in the form

$$
\omega^{2} x^{\prime \prime}+x=\varepsilon\left\{-\gamma x^{3}+h\left(1-x^{2}\right) \omega x^{\prime}\right\},
$$

where primes denote differentiation with respect to $\tau ; \omega$ is the unknown frequency; $\varepsilon$ is a formal parameter indicating the smallness of the right hand side.

The problem of interest is to determine the frequency of stationary self-excited oscillation satisfying initial condition

$$
\left.x^{\prime}(\tau)\right|_{\tau=0}=x^{\prime}(0)=0 .
$$

According to the standard Poincaré method, both unknowns $x$ and $\omega$ are expanded in powers of $\varepsilon$, that is

$$
\begin{aligned}
& x=x_{0}(\tau)+\varepsilon x_{1}(\tau)+\varepsilon^{2} x_{2}(\tau)+\ldots, \\
& \omega=1+\varepsilon \omega_{1}+\varepsilon^{2} \omega_{2}+\ldots
\end{aligned}
$$

Corresponding to the initial condition $(2.2)$, those of $x_{0}, x_{i}(i=1,2, \ldots)$ are

$$
x_{0}^{\prime}(0)=0, \quad x_{i}^{\prime}(0)=0 \quad(i=1,2, \ldots) .
$$

Substituting (2.3), (2.4) into (2.1), equating the terms of like powers of $\varepsilon$ yield

$$
\begin{aligned}
& x_{0}^{\prime \prime}+x_{0}=0, \\
& x_{1}^{\prime \prime}+x_{1}=-2 \omega_{1} x_{0}^{\prime \prime}-\gamma x_{0}^{3}+h\left(1-x_{0}^{2}\right) x_{0}^{\prime}, \\
& x_{2}^{\prime \prime}+x_{2}=-2 \omega_{1} x_{1}^{\prime \prime}-\left(\omega_{1}^{2}+2 \omega_{2}\right) x_{0}^{\prime \prime}-3 \gamma x_{0}^{2} x_{1} \\
& \quad+h\left(1-x_{0}^{2}\right)\left(x_{1}^{\prime}+\omega_{1} x_{0}^{\prime}\right)-2 h x_{0} x_{1} x_{0}^{\prime},
\end{aligned}
$$

With regard to the initial condition $x_{0}^{\prime}(0)=0$, the general solution of the differential equation $(2.6)$ is

$$
x_{0}=a_{0} \cos \tau, \quad a_{0}=\text { const. }
$$

Using (2.9), the differential equation (2.7) becomes

$$
\begin{array}{r}
x_{1}^{\prime \prime}+x_{1}=2 \omega_{1} a_{0} \cos \tau-\gamma a_{0}^{3}\left(\frac{3}{4} \cos \tau+\frac{1}{4} \cos 3 \tau\right) \\
-h a_{0}\left(1-\frac{a_{0}^{2}}{4}\right) \sin \tau+\frac{1}{4} h a_{0}^{3} \sin 3 \tau .
\end{array}
$$

To eliminate secular terms in $(2.10)$, the unknowns $a_{0}$ and $\omega_{1}$ should be taken as:

$$
a_{0}=2, \quad \omega_{1}=\frac{3}{8} \gamma a_{0}^{2}=\frac{3}{2} \gamma .
$$


Then

$$
\begin{aligned}
& x_{1}^{\prime \prime}+x_{1}=-\frac{\gamma a_{0}^{3}}{4} \cos 3 \tau+\frac{h a_{0}^{3}}{4} \sin 3 \tau=-2 \gamma \cos 3 \tau+2 h \sin 3 \tau, \\
& x_{1}=\frac{\gamma}{4} \cos 3 \tau-\frac{h}{4} \sin 3 \tau+a_{1} \cos \tau+b_{1} \sin \tau,
\end{aligned}
$$

where $a_{1}, b_{1}$ are constants to be determined.

To satisfy the condition (2.5) for $i=1, b_{1}$ should be taken

$$
b_{1}=\frac{3 h}{4} \text {. }
$$

With regard to $(2.9),(2.11),(2.13),(2.14)$, the differential equation $(2.8)$ can be simplified as:

$$
x_{2}^{\prime \prime}+x_{2}=\left(4 \omega_{2}-6 \gamma a_{1}+\frac{15 \gamma^{2}+h^{2}}{4}\right) \cos \tau+h\left(2 a_{1}+\gamma\right) \sin \tau+\ldots,
$$

where non-written terms do not contain the first harmonics.

Eliminating secular terms in (2.15) gives

$$
a_{1}=-\frac{\gamma}{2}, \quad \omega_{2}=\frac{-1}{16}\left(2 \gamma^{2}+h^{2}\right)
$$

Thus, the first two formulas for frequency are:

$$
\begin{aligned}
\omega_{I} & =1+\frac{3 \gamma}{2} \\
\omega_{I I} & =1+\frac{3 \gamma}{2}-\frac{27 \gamma^{2}+h^{2}}{16} .
\end{aligned}
$$

Preliminary remark: for large $\gamma$, the frequency $\omega_{I I}$ becomes negative $\left(\omega_{I I}=0\right.$ if $\gamma \approx 1.3333 \ldots$ ); this announces that the frequency obtained from the standard Poincaré method is not reliable for large $\gamma$.

\section{Frequency from a modified Poincaré method}

In this section, the problem of interest is treated by the modified Poincare method as presented in [2].

Assuming that the strongly nonlinear Vanderpol oscillator considered is near certain linear one of unknown frequency $\omega$, we rewrite the differential equation (1.1) as:

$$
\ddot{x}+\omega^{2} x=\mu\left\{\left(\omega^{2}-1\right) x-\gamma x^{3}+h\left(1-x^{2}\right) \dot{x}\right\},
$$

or, with the new time $\tau=\omega t$ :

$$
\omega^{2}\left(x^{\prime \prime}+x\right)=\mu\left\{\left(\omega^{2}-1\right) x-\gamma x^{3}+h\left(1-x^{2}\right) \omega x^{\prime}\right\}
$$


where $\mu$ is a small formal parameter introduced to indicate the smallness of the right hand side.

Both unknowns $x$ and $\omega$ are now expanded in powers of $\mu$, that is:

$$
\begin{aligned}
& x=x_{0}(\tau)+\mu x_{1}(\tau)+\mu^{2} x_{2}(\tau)+\ldots \\
& \omega=\omega_{0}+\mu \omega_{1}+\mu^{2} \omega_{2}+\ldots
\end{aligned}
$$

where $\omega_{0}$ is the approximate frequency of order $\mu^{0}$ (not 1 as in the standard Poincaré method)

Substituting (3.3), (3.4) into (3.2), equating the terms of like powers of $\mu$ yield

$$
\begin{aligned}
\omega_{0}^{2}\left(x_{0}^{\prime \prime}+x_{0}\right)=0 & \\
\omega_{0}^{2}\left(x_{1}^{\prime \prime}+x_{1}\right)= & \left(\omega_{0}^{2}-1\right) x_{0}-\gamma x_{0}^{3}+h\left(1-x_{0}^{2}\right) \omega_{0} x_{0}^{\prime}, \\
\omega_{0}^{2}\left(x_{2}^{\prime \prime}+x_{2}\right)= & -2 \omega_{0} \omega_{1}\left(x_{1}^{\prime \prime}+x_{1}\right)+\left(\omega_{0}^{2}-1\right) x_{1}+2 \omega_{0} \omega_{1} x_{0}-3 \gamma x_{0}^{2} x_{1} \\
& \quad+h\left(1-x_{0}^{2}\right)\left(\omega_{0} x_{1}^{\prime}+\omega_{1} x_{0}^{\prime}\right)-2 h x_{0} x_{1} \omega_{0} x_{0}^{\prime},
\end{aligned}
$$

The general solution $x_{0}$ satisfying the initial conditions $x_{0}^{\prime}(0)=0$ is

$$
x_{0}=a_{0} \cos \tau, \quad a_{0}=\text { const. }
$$

Substituting (3.8) into (3.6) gives:

$$
\begin{aligned}
\omega_{0}^{2}\left(x_{1}^{\prime \prime}+x_{1}\right)= & \left(\omega_{0}^{2}-1\right) a_{0} \cos \tau-\gamma a_{0}^{3}\left(\frac{3}{4} \cos \tau+\frac{1}{4} \cos 3 \tau\right) \\
& -h \omega_{0} a_{0}\left(1-\frac{a_{0}^{2}}{4}\right) \sin \tau+\frac{h \omega_{0} a_{0}^{3}}{4} \sin 3 \tau .
\end{aligned}
$$

To eliminate secular terms in (3.9), $a_{0}$ and $\omega_{0}$ should be taken as

$$
a_{0}=2, \quad \omega_{0}^{2}=1+\frac{3 \gamma}{4} a_{0}^{2}=1+3 \gamma
$$

Using (3.8), (3.10), the differential equation governing $x_{1}$ becomes

$$
\omega_{0}^{2}\left(x_{1}^{\prime \prime}+x_{1}\right)=-\frac{\gamma a_{0}^{3}}{4} \cos 3 \tau+\frac{h \omega_{0} a_{0}^{3}}{4} \sin 3 \tau=-2 \gamma \cos 3 \tau+2 h \omega_{0} \sin 3 \tau
$$

and the general solution $x_{1}$ is:

$$
x_{1}=\frac{\gamma}{4 \omega_{0}^{2}} \cos 3 \tau-\frac{h \omega_{0}}{4 \omega_{0}^{2}} \sin 3 \tau+a_{1} \cos \tau+b_{1} \sin \tau,
$$

where $a_{1}, b_{1}$ are two constants to be determined. 
To satisfy the initial condition (2.5) for $i=1, b_{1}$ should be taken as

$$
b_{1}=\frac{3 h_{0} \omega_{0}}{4 \omega_{0}^{2}}
$$

The differential equation (3.7) can be simplified as:

$$
\begin{aligned}
\omega_{0}^{2}\left(x_{2}^{\prime \prime}+x_{2}\right)=h \omega_{0}\left(2 a_{1}+\frac{\gamma}{\omega_{0}^{2}}\right) \sin \tau+\left(4 \omega_{0} \omega_{1}-6 \gamma a_{1}-\frac{3 \gamma^{2}+6 h^{2} \omega_{0}^{2}}{4 \omega_{0}^{2}}\right) \cos \tau \\
+(\text { higher harmonic terms })
\end{aligned}
$$

Eliminating secular terms in (3.14) gives:

$$
a_{1}=-\frac{\gamma}{2 \omega_{0}^{2}}, \quad \omega_{1}=-\frac{9 \gamma^{2}+h^{2} \omega_{0}^{2}}{16 \omega_{0}^{3}} .
$$

Thus, the two formulas for frequency are

$$
\begin{aligned}
\omega_{I *} & =\omega_{0}=\sqrt{1+3 \gamma}, \\
\omega_{I I *} & =\omega_{0}+\omega_{1}=\sqrt{1+3 \gamma}\left\{1-\frac{9 \gamma^{2}+h^{2}(1+3 \gamma)}{(1+3 \gamma)^{2}}\right\},
\end{aligned}
$$

or, by neglecting $h^{2}$ (if $\gamma$ is large enough) we have

$$
\omega_{I I *}=\sqrt{1+3 \gamma}\left\{1-\frac{9 \gamma^{2}}{(1+3 \gamma)^{2}}\right\} .
$$

\section{Comparison and conclusion}

For $h=0.05$ and for different values $\gamma$, using (2.17) and (3.16), (3.17), approximate frequencies $\omega_{I}, \omega_{I I}$ (from the standard Poincaré method) and $\omega_{I *}, \omega_{I I *}$ (from the modified Poincaré method) are shown in the third, fourth and fifth, sixth columns, of the table standing at the end of this article, respectively. The frequencies $\omega_{c}$ in the second column are obtained by analyzing the amplitude spectrum [3] of stationary self-excited oscillations $x(t)(t \in[0,12 \pi]$, number of times $N=2048)$. It can be seen that, the first approximate frequencie $\omega_{I}$ obtained from the standard Poincaré method can be used only for $\gamma \ll 1$ (for $\gamma=1$, the relative error is $20 \%$ ), the second approximation $\omega_{I I}$ must be rejected. On the contrary, the frequencies $\omega_{I *}, \omega_{I I *}$ obtained from the modified Poincare method are practically acceptable and $\omega_{I I *}$ is better than $\omega_{I *}$ (for $\gamma=20$, the relative error of $\omega_{I I *}$ is of order $2.2 \%$ ). 
Table 1

\begin{tabular}{cccccc}
\hline$\gamma$ & $\omega_{c}$ & $\omega_{I}$ & $\omega_{I I}$ & $\omega_{I *}$ & $\omega_{I I *}$ \\
\cline { 7 - 7 } \cline { 6 - 7 } & 1.0000 & 1.0000 & 0.9999 & 1.0000 & 0.9999 \\
1 & 2.0000 & 2.5000 & 0.1324 & 2.0000 & 1.9296 \\
2 & 2.5000 & 4.0000 & - & 2.6457 & 2.5342 \\
3 & 3.0000 & 5.5000 & - & 3.1623 & 3.0021 \\
4 & 3.5000 & 7.0000 & - & 3.6055 & 3.4450 \\
5 & 3.8333 & 8.5000 & - & 4.0000 & 3.7802 \\
6 & 4.1666 & 10.0000 & - & 4.3589 & 4.1144 \\
7 & 4.5000 & 11.5000 & - & 4.6904 & 4.4233 \\
8 & 4.8333 & 13.0000 & - & 5.0000 & 4.7120 \\
9 & 5.1666 & 14.5000 & - & 5.2915 & 4.9840 \\
10 & 5.3333 & 15.0000 & - & 5.5678 & 5.2418 \\
12 & 5.8333 & 19.0000 & - & 6.0828 & 5.7228 \\
14 & 6.3333 & 22.0000 & - & 6.5574 & 6.1664 \\
16 & 6.6666 & 25.0000 & - & 7.0000 & 6.5802 \\
18 & 7.1666 & 28.0000 & - & 7.4162 & 6.9694 \\
20 & 7.5000 & 31.0000 & - & 7.8102 & 7.3380 \\
\hline
\end{tabular}

This publication is completed with the financial support from The Council for Natural Science of Vietnam.

\section{REFERENCES}

1. Nayfeh A. N. Pertubation method, Wiley, New York, 1973.

2. Nguyen Van Dinh. The Poincaré method for a strongly nonlinear Duffing oscillator. Vietnam Journal of Mechanics, NCST of Vietnam Vol.25, 2003, No 1 (19-25).

3. Bendat J. S., Piersol A. G. Engineering applications of correlation and spectral analysis. Wiley-Inter Science, New York, 1980.

Received April 15, 2003

\section{TẦN SỐ CƯA CHẤN TƯ VAN-DER-POL CÓ PHI TUYẾN HỒI PHỤC MẠNH BẬC BA}

Khảo sát chấn tử Vanderpol có phi tuyến hồi phục mạnh bậc ba và kích động tự chấn yếu. Xét quan hệ giữa tần số của chế độ tự chấn dừng với hệ số phi tuyến hồi phục. Phương pháp chuẩn Poincaré không áp dụng được nên một biến thể của nó trình bày ở [2] được sử dụng. Kết quả ở xấp xì thứ hai thực tế chấp nhận được. 